\title{
ESAS PAPITAS ME ESTÁN MIRANDO! SILVIA RIVERA CUSICANQUI Y LA TEXTURA CH'IXI DE LOS MUNDOS ${ }^{1}$
}

\author{
Francisco Pazzarelli
}

\section{Mestiz*s}

De algún modo, las formas del encuentro evocado en los párrafos que siguen fueron montándose en los días y momentos previos al encendido del grabador ${ }^{3}$. Habíamos intercambiado ya algunos emails antes de su viaje y luego en Córdoba, donde ella dictaba su curso. Todavía no sabíamos si habría tiempo disponible para conversar, entonces aproveché a preguntarle algunas cosas durante sus clases y adelantar algunas ideas en los recreos. El jueves previo a la entrevista, nos encontramos casualmente en el campus de la universidad mientras nos dirigíamos a escuchar su conferencia y acordamos conversar al otro día.

Ese viernes fue particularmente caluroso y húmedo. Después del mediodía, salimos con ella y otros estudiantes en busca de un algún lugar para comer. En el camino, cambiamos de planes y pasamos por la Feria del libro, que estaba desarrollándose en la ciudad, para buscar unas obras de Fogwill. No tuvimos éxito y optamos por hacer fotocopias para que pudiera llevarlas a La Paz. Luego de unas vueltas más, llegamos a un buffet vegetariano, atendido por sus dueños de origen coreano. Fuimos a comer con nuestras bandejas al primer piso, donde el calor y los vapores de la cocina nos hacían compañía. Una de las estudiantes, dijo que tenía algo para revitalizar la comida y sacó de su mochila varios locotos, ajíes picantísimos de distintos colores,

\footnotetext{
${ }^{1}$ Silvia Rivera Cusicanqui es socióloga, ensayista y fue docente en la Universidad Mayor de San Andrés, en La Paz, Bolivia. Se reconoce como una eterna maestra de oficio y artesana intelectual. Ha dictado cursos y seminarios en diferentes universidades del mundo. De su autoría, se resalta el ya clásico Oprimidos pero no vencidos. Luchas del campesinado aymara y quechwa 1900-1980 (1986). Recientemente, ha publicado Sociología de la Imagen. Miradas ch'ixi desde la historia andina (2015) y Mito y Desarrollo en Bolivia. El giro colonial del gobierno del MAS (2015).

Esta entrevista fue originalmente realizada para formar parte del Dossier "Contradiscursos Afroindígenas sobre Mistura, Sincretismo e Mestiçagem. Estudos Etnográficos", organizado por Marcio Goldman, Marcela Coelho de Souza, Julia Sauma, María Belén Hirose y Francisco Pazzarelli, de pronta aparición.

${ }^{2}$ Argentina. Antropólogo, investigador del Instituto de Antropología de Córdoba - CONICET y profesor asistente en la Universidad Nacional de Córdoba, Argentina. Realiza su trabajo etnográfico en comunidades indígenas de las regiones de quebrada y puna de los Andes jujeños, en el noroeste argentino, indagando sobre socialidad, culinaria y relaciones de predación.

${ }^{3}$ A excepción de esta, todas las notas que siguen corresponden a agregados de SRC (o sugeridos por ella), realizados con la intención de esclarecer alguna postura o brindar traducciones aproximadas de los términos aymara. Agradezco especialmente a Rosa Quiroga por haberme acompañado y aconsejado en varios de los paseos que supusieron estas conversaciones.
} 
envueltos en una servilleta. Los compartió y la comida se tornó inmediatamente en una fusión por demás sabrosa. Silvia aprovechó para contarnos sobre el picor de los ajíes que ella misma cosecha con sus estudiantes y rememoró algunas anécdotas sobre la casa que habitan y utilizan todos ellos en La Paz. Mientras, a la temperatura del día y los vapores de la cocina, nosotros sumábamos algunas gotas de sudor que nos regalaban los locotitos.

Silvia Rivera Cusicanqui nunca deja de evocar y propiciar el calor de la reunión y de lo colectivo como potencia predilecta y lugar privilegiado desde donde pensar. Lo hace cuando habla sobre la Colectivx Ch'ixi a la que pertenece, con sede en el barrio de Tembladerani en La Paz. Lo hace cuando rememora el Taller de Historia Oral Andina, que desde los '80 se ocupó de rescatar las memorias y experiencias de resistencia indígena y obrera en Bolivia. Lo hace también cuando evita presentarse a sí misma solo como una ensayista o socióloga. Ella es en primer lugar miembro de un colectivo y reivindica a cada momento la importancia del encuentro entre personas con diferentes trayectorias e intereses. Siempre se piensa con otros, siempre es mejor cuando las cosas se entreveran.

Silvia posee un gusto difícil de describir sobre aquello que está mezclado, superpuesto. Lo deja evidente en sus libros y en sus clases, claro. Pero también al caminar por la calle, cuando se detiene a preguntar sobre tal o cual edificio antiguo intervenido por la modernidad cordobesa o al elegir las comidas que combina en el plato. La mezcla se le aparece como un paño que debe ser radiografiado. Pero no para diseccionarlo, aclara; ni para analizar quirúrgicamente qué parte le pertenece a qué todo. Más bien, todo parece pasar como si le interesara revitalizar la potencia de las costuras haciendo los hilos más visibles: sin descoserlos, apreciando los intervalos, los espesores y las superficies rugosas de junturas y nudos.

Después del picor de la comida, comimos un flan que ofrecían en unas bandejitas de plástico. Como el salón estaba vacío -el buffet ya estaba cerrando- nos mudamos junto a la ventana, mientras yo encendía el grabador y ella me preguntaba, entonces, sobre qué cosas yo todavía quería charlar después de haber conversado tanto.

Francisco Pazzarelli (FP): Me interesa especialmente entender los caminos de tus últimos años, de lo que también se ha publicado aquí en Argentina. ¿Cuál sería una buena manera de introducirnos a tu análisis de lo ch’ixi? Si quisiéramos rastrear la genealogía de estas proposiciones, ¿a partir de cuáles elementos vos dirías que comenzaste a pensarlo? 
Silvia Rivera Cusicanqui (SRC): El interés por lo ch'ixi está relacionado con lo que sería la ideología oficial del mestizaje, la confluencia armónica de los dos polos, el español y el indio, que daría lugar a esa fusión imaginaria: la raza cósmica (en el caso de México), o al contrario, la raza vencida y degenerada de Arguedas o Moreno, que reuniría lo peor ¿no es cierto? En esta operación maniquea, la naturaleza de la mezcla se soslaya; tácitamente se asume una dominante blanca. El proceso o la deriva de esta mezcla siempre es hacia el blanqueamiento. No se imagina ni tolera un proceso inverso, o quizás se lo desprecia aún más porque sería una especie de regresión voluntaria.

El segundo efecto de ello es que ambas versiones pretenden borrar la huella de la contradicción que dio origen al deseo de identificación con un tercero... se la borra. Es una dominación que borra su propia huella. Y eso ha sido lo 'conciliador' de la política oficial del mestizaje, que además es una política del olvido, se basa en el olvido de la contradicción. El hecho es que hay formas, digamos, de desdoblamiento, porque finalmente hay un dualismo intrínseco a la relación colonial, que hace que el mestizo termine siendo, como dicen en Chile, "mestizo al derecho" o "mestizo al revés", el chileno y el "roto". En Bolivia es el mestizo versus el cholo. Pero en vez de verse al cholo como uno de los posibles destinos de esa categoría - que en vez de acercarse a lo blanco, desee o quiera ser más "indio" - tal opción no es valorada. Se la ve como un proceso trunco de blanqueamiento. El cholo no pudo llegar a ser mestizo, no pudo blanquearse porque 'se le salió el indio', porque lo 'traicionó la sangre', o sus hábitos, o el color, o sus promiscuidades. Es decir, es igualmente un proceso de blanqueamiento sólo que uno fue exitoso -mestizo- y otro frustrado -cholo. Entonces lo cholo nunca se ve como opción, como posibilidad afirmativa, ¿ya? Por eso es que siendo que mestizo y cholo te dan la posibilidad de un mestizo que no sea blanqueado, en la choledad realmente existente ${ }^{4}$ hay como un deseo incumplido de blanqueamiento, en lugar de ser la suya una identificación afirmativa. Muchas veces se ha reflexionado sobre la conversión del 'estigma' en 'emblema': "black is beautiful” Goffman. O sea, si te han jodido porque eres negro, tú afirmas que lo negro es hermoso. Como dicen que dijo una lideresa aymara de las rebeliones de principios del siglo pasado: "si nos han

\footnotetext{
${ }^{4}$ Excepción hecha de las cholas y cholos anarquistas de la FOL (Federación Obrera Local), la FAD (Federación Agraria Departamental) y de muchas articulaciones pragmáticas de la choledad que se dan en la actualidad. Ver al respecto la compilación "Anarquismo ayer y hoy", en la revista El Colectivo 2, número 2; y Rivera Cusicanqui y Lehm Ardaya, 2014.
} 
oprimido como indixs, como indixs nos liberaremos". Pero nadie, o muy pocos, han dicho "yo soy cholo'5; no se ve lo cholo como un espacio de afirmación, de alteridad, como al decir 'somos a la vez modernxs y ancestrales, somos urbanxs, urbandinxs. Somos cholxs!'.

Aunque, por ejemplo, René Zavaleta, en sus análisis sobre la revolución de 1952, habla de ella como una 'revolución de los cholos', como la instauración de una nueva forma de ser boliviano que no es la de la pretendida blanquitud oligárquica. Nadie le ha dado pelota a este concepto de lo cholo; Zavaleta muestra que tiene mucho de ch'ixchi, el equivalente qhichwa de ch'ixi, porque intercala en sus textos conceptos en qhichwa que no han llamado la atención de sus exégetas. Creo que su potencia teórica, sobre todo en su fase marxista (de Zavaleta), ha eclipsado sus otros esfuerzos conceptuales, más cercanos a un pensamiento descolonizador, que aparecen ya esbozados en su obra póstuma sobre lo nacional y popular en Bolivia. Luis Tapia vislumbra algo de ello en su tesis doctoral sobre Zavaleta, en cuya obra póstuma se hace explícito el protagonismo indio en la historia de Bolivia. Es curioso entonces cómo se lo ha blanqueado a Zavaleta. En el libro que estoy escribiendo tengo un breve capítulo destinado a conectar la noción de 'abigarramiento' con la de lo ch'ixi, creo que ya es hora de releer a Zavaleta desde el akapacha ${ }^{6}$ de la presente crisis, reconsiderar la historia que él interpreta, pero haciéndolo desde otras lógicas y con otras alegorías, para hallar vetas descolonizadoras en el mestizaje ch'ixi. Y claro, la nueva generación del indianismo katarista lamentablemente ha volteado en bloque la relectura de Zavaleta, creen que toda persona que se reconozca como mestiza no tiene nada que decirles en ese terreno crítico que es la descolonización.

FP: No habría nada de bueno en lo mestizo...

SRC: No, nada y es el 'enemigo'. Es una tontería. Yo les he dicho a los indianistas: sería un suicidio colectivo si decidieran hacer política y negaran la posibilidad de descolonización del mestizaje, lo mismo que si negaran la posibilidad de la liberación femenina. Descolonizar el mestizaje, en primer lugar, es arrebatarlo del destino de conformismo que lo subyuga como un sector de vocación estatal, masculina y centrista. O sea, la centralidad estatal como el anhelo mestizo; la patria mestiza sería la patria del Estado centralizado, fuerte, con mayúscula. Es

\footnotetext{
${ }^{5}$ Un ejemplo notable de esta postura afirmativa es el trabajo de Gutiérrez Flores (2010).

${ }^{6}$ En aymara, refiere a este tiempo/espacio, aquél que corresponde al aquí y ahora.
} 
evidente que para mí ésta es la versión colonizada del mestizaje, que no reconoce su propia huella y niega su propio nombre. El epítome de esta construcción cultural es el mestizaje colonial cochabambino. Es notable la identificación de lo mestizo con el Estado, explícitamente en el caso de Carlos Montenegro y Augusto Céspedes e implícita y solapadamente en Álvaro García Linera. Todos lo asumen como una forma universal de la ciudadanía y niegan la huella colonial que constituye al ser mestizo. En el libro "Identidad Boliviana. Nación, mestizaje y plurinacionalidad", de García Linera (García Linera, 2014), se borra la noción misma de mestizaje, se la despacha sin contemplaciones, tan sólo para revestirla y travestirla como identidad boliviana. Qué duda cabe, la intelligentsia del cholaje cochabambino se ha especializado en condimentar y ornamentar la supuesta "identidad boliviana" con algunas pinceladas indias para capitalizar la plusvalía simbólica de la indianidad en favor de su proyecto capitalista y mestizo (valga la redundancia) ${ }^{7}$.

En la última compilación del pensamiento crítico boliviano que hicimos con Vicky Aillón (Rivera Cusicanqui y Aillón Soria, 2015), hacemos un análisis de lo cochabambino como metáfora de la centralidad, y mostramos cómo estas pulsiones de centralidad han terminado articulando o pretenden articular la totalidad social boliviana en un proyecto concebido desde un fragmento muy pequeño de la población, que es esta élite mestiza, reducto de un mestizaje colonial. Eso hace que se nieguen otras formas y que se produzca una especie de eclipse sobre la choledad como un hijo de mestizo que pudiera tener algo que decir en términos de descolonización. O sea, descolonizar lo cholo es desligarlo de todas las tendencias a subsumirlo en modos de consumos posmodernos 'pluri-multis', que deje de ser el espectáculo que es, indianizado, para aparecer en las entradas folclóricas; que deje de ser expropiado de su plusvalía simbólica, cuando la ropa de la chola se convierte en emblema de lo étnico en Bolivia; cuando el sombrero italiano termina en la sala del turista gringo representando la indianidad boliviana. Incluso pocos italianos van a reconocer que el sombrero de la chola está a la vuelta de la esquina en un pueblito de su país llamado Borsalino. Todas esas paradojas hacen que las llamadas 'tradiciones indígenas' se conviertan en ornamentos estatales y folclóricas, bloqueando así el juego y el proceso de las identificaciones étnicas y su potencial descolonizador.

\footnotetext{
${ }^{7}$ No en vano, el patujú (una de las flores nacionales de Bolivia que crece en las tierras bajas) es el símbolo favorito del vicepresidente García Linera, autor del mencionado libro ("La identidad boliviana..."), cuya distribución gratuita ha sido seguramente financiada con la plata del gas. Ver al respecto: Rivera Cusicanqui, 2015. 


\section{Manchad*s}

Mientras conversábamos, Silvia observa una pulsera ll'oque en mi muñeca. La había recibido unos meses antes durante mi trabajo de campo en Jujuy, de parte de una familia amiga. Un artefacto de hilos blancos y negros, 'manchado', tejido al 'revés', que se utiliza para sobrevivir a los males de agosto, para protegerse. Similar a las hondas tejidas para atacar a los enemigos en las batallas. Me señala que eso es exactamente lo que me está queriendo decir. Y comienza a enumerar en aymara una serie de nombres de animales con diseños análogos a los de mi pulsera.

El barullo de los autos sube hacia las ventanas del comedor y se suma al de algunas conversaciones en coreano de los cocineros que están en planta baja. No entiendo aymara y acerco mi oído justo cuando Silvia retoma el español. Pienso que el grabador no está, ni remotamente, captando el fragor del momento. Pero ella repite sus palabras y vuelve a explicar: quiere decirme que tenemos que conectarnos con las formas y los modos que reclama mi pulsera. Que se trata de pensar con el ll'oque y no solo sobre él. Pensar con la potencia que encierran los gestos técnicos de los hilos torcidos al revés, que al envolverse sobre sí mismos convocan con ese movimiento a los otros del mundo. Que entre el hilo blanco y el negro existe un intervalo que no está 'vacío'. Inspira profundamente y me mira fijo, como preguntando si entendí.

SRC: En el libro "Principio Potosí Reverso" (Rivera Cusicanqui y el Colectivo, 2010) con mi grupo -hoy Colectivx Ch'ixi- trabajamos mucho intentando entender las abigarradas prácticas populares en torno a las fiestas de los santos patronos. Para ello partimos de los conceptos aymaras que escuchábamos, pero intentando ir más allá de sus usos pragmáticos. Por eso convertimos a algunos de ellos en conceptos-metáfora, tomado de Spivak, en el esfuerzo de restituir sus niveles abstractos y hermenéuticos, sus niveles teóricos, ¿ya? Lo hacíamos de manera tal que pudiéramos imaginar otros modos de pensar, teniendo como base la polisemia de cada concepto, porque el aymara es una lengua que se presta para eso. Sobre todo porque es aglutinante y se pueden aprovechar las diferentes lógicas combinatorias que hay en sus sufijos. De ese modo intentamos pensar a partir de las hilachas que le quedan al idioma en sus múltiples usos y que pueden permitir ir más allá de esos niveles pragmáticos.

Uno de estos conceptos, lo ch'ixi, es por ejemplo lo que Victor Sapana, un escultor aymara, relaciona con los animales poderosos. Son ch'ixis porque son a la vez negros y blancos, o de un colorido manchado. Eso es lo que hace su condición indeterminada, es lo que les brinda 
su fuerza explosiva. Porque, por ejemplo, la serpiente es de arriba y es de abajo, es masculina y es femenina, es lluvia pero a la vez es veta de metal, se simboliza como rayo que cae de lo alto, pero también como fuerza subterránea. Toda esa dimensión metafórica compleja explica por qué los rebeldes andinos eligieron llamarse kataris y amarus, 'serpiente' en aymara y qhichwa respectivamente. Y explica por qué el emblema, el objeto que mejor traduce la condición torcida y dual del katari, es la q'urawa, la honda andina que se ha utilizado eficazmente en las movilizaciones y bloqueos de caminos de 2000-2005.

FP: La honda generalmente también es 'manchada' cuando se teje.

SRC: Exacto. Y es además tejida con lana torcida al revés, que es una forma de revertir, es ch 'iqa ch'anka, y eso es lo que revierte el mal. La lana o hilo ch 'iqa ch'anka está torcida al revés, y eso permite contrarrestar el daño: en palabras de don Víctor, la imagen de la serpiente 'sirve para defendernos de la maldad del enemigo'. Hay una fuerza material efectiva, que en la q'urawa se duplica o suplementa con la imagen de la serpiente. Pero además hay muchos otros animales ch'ixi: jamp'atu o sapo, jararanku o lagarto, kusi-kusi o araña, un inventario de animales poderosos, animales misteriosos digamos, que nos permiten transitar entre opuestos. Todo eso para mí tiene una potencia teórica impresionante.

FP: Es que permiten alguna especie de ‘tránsito’ entre mundos.

SRC: Sí, pero no como mediación ni tampoco como conciliación de opuestos. No es síntesis, ni es hibridación, mucho menos fusión. Se mantienen esos opuestos y para mí el gesto descolonizador consistiría en rescatarlos de los envoltorios capitalistas, consumistas y alienantes a los que la historia del capital los ha condenado: el liberalismo, el multiculturalismo estatal y del Banco Mundial, el reformismo, etc. Despojarlos y desnudarlos para descubrir en su pureza y en su fuerza una energía de descolonización que permita sacudir las estructuras de lo heredado. Se trata de un gesto que, por ejemplo, nos lleva a reconocer qué es lo que tenemos de Europa, algo de lo que podamos hacernos cargo. Podríamos enumerar muchas cosas, comenzando por el castellano (como lengua materna o adquirida), pero yo pienso que la idea de libertad individual puede vincularse con algo de esa episteme que yo llamo 'noratlántica' porque sin duda surge al 
calor de las luchas anarquistas, de las luchas del mundo del trabajo. La idea de derechos, la idea de igualdad... yo diría que son ideas ligadas a la 'equivalencia' más que a la igualdad. No se trata de homogeneización, sino de equivalencia, sería como decir 'tu forma de conocer es equivalente a la mía'; aunque sean muy distintas en su episteme, en sus conceptos, etc, son equivalentes. Son igualmente necesarias. Quizás podríamos ahondar en esta episteme acudiendo a la noción de homo faber... el mundo tecnológico, esa mente analítica que busca siempre desarmar lo que existe para entenderlo... Claro que esta mentalidad puede llegar a ser destructora si se exacerba, si se convierte en la única modalidad. Es lo que pasa con la ciencia hegemónica, que puede llegar a ser absolutamente devastadora. Pero los niños nos muestran esa pulsión analítica en su estado primordial, cuando desarman un juguete para saber cómo funciona, están expresando un deseo de conocimiento. Quizás esta idea no sea localizable en una determinada episteme, pero sobre todo creo que nos permite pensar en la reconexión de la mano con el cerebro, en la recualificación del hacer, por encima de otros gestos.

\section{$\operatorname{Otr}^{*}{ }_{S}$}

Aunque sin olvidar los locotitos de aquella tarde de septiembre, la edición del texto final de esta entrevista gozó de algunos intervalos que la aplazaron un poco. Primero, por causa de algunas superposiciones de tareas que incluían, entre otras cosas, una campaña de la Colectivx en contra de la minería en Teoponte, algo de lo que yo me había anoticiado en internet. Segundo, porque Silvia estaba ocupada trabajando y cocinando para las almas de sus muertos, que llegaban a comer en los primeros días de noviembre; incluso, me envió una foto por email para contarme sobre la envergadura del trabajo. La revisión final, en cambio, se adelantó: dos días antes del nuevo plazo el archivo "Final" estaba en mi correo. Eso porque la Colectivx iniciaba al otro día su temporada de siembra que iba a tener ocupados a todos sus miembros, la protagonista de esta entrevista incluida.

Lo ch'ixi no es solo algo sobre lo que se piensa sino también algo a través de lo que se puede pensar; por lo que nunca se desprende del compromiso con el hacer. Lo ch'ixi supone e implica una suerte de haceres que no pueden ser descriptos sin involucrarse activamente en ellos. Cocinando, sembrando o haciendo carteles de protesta. Como si hubiera primero que aprender a convivir con lo ch'ixi y sus formas antes de poder hablar de o sobre él. O como ella misma pregunta: ‘cómo vivirán los que hablan tanto del vivir bien?' 
La preocupación por el hacer reclama, Silvia lo enfatiza siempre, una cierta impaciencia por recualificar a la actividad intelectual como artesanía, llevando a serio las formas creativas que ello puede suponer. Formas que ni se reducen a un bagaje previo y limitado de herramientas ni se definen en un manojo escaso de resultados posibles. La creatividad, así, no se presenta solo como un medio sino como el lugar hacia donde deberían tender nuestros esfuerzos. Sin nunca perder de vista, claro, que ninguno de esos esfuerzos son sólo 'nuestros': ellos solo existen si están contaminados, sucios, manchados y parcialmente habitados por otros.

Es en este sentido que las almas de los muertos, la tierra amenazada por la minería y las semillas de noviembre se revelan en la edición de esta entrevista. Cada uno de estos intervalos hablan menos de agendas apretadas que de los devenires propios de un texto -de todos los textos?- que está siempre e inevitablemente intervenido por otros. Tal vez fueron aquellos otros convocados al hablar del hilo torcido de las q'urawas. O tal vez aparecieron solos, porque querían. Lo cierto es que penetran con fuerza, se hacen lugar y generan intervalos fértiles que permiten pensar, que habilitan el hacer. Y es con ellos que los mundos, y los textos, ganan existencia y espesor.

FP: Hay una relación interesante, compleja, que vos siempre mencionás entre el 'trabajo' y el 'hacer', o entre el 'trabajo' y la 'actividad'. Y allí una cuña de raigambre anarquista que sirve para pensar algunas cosas, no?

SRC: Absolutamente, y además ahí yo creo que hay ciertas variantes anarquistas del 'no trabajo', que es como el equivalente del 'no trabajo infantil'. Yo diría mejor no a la explotación, ni infantil, ni masculina, ni femenina, ni adulta. La explotación es lo que está mal. Pero el trabajo...

FP: El trabajo como actividad, como hacer...

SRC: Que la actividad, que el hacer sea denigrado en favor del ocio, ¡eso ya parece bíblico! Porque entonces ¿el trabajo sería un castigo? No. La idea es reinjertar el trabajo en la fiesta, en la convivencia, en el goce, en el ritual. Y un poco es lo que se muestra en el video ${ }^{8}$ : el maestro dice

\footnotetext{
${ }^{8}$ Video de la autoconstrucción del Tambo en La Paz, espacio levantado materialmente los integrantes de la Colectivx para albergar sus cursos y talleres libres. 
'no, no se puede trabajar en construcción todo el día. También hay que charlar, akhullikar', sembrar'. Reivindica la pluralidad de actividades, no ese trabajo tedioso que consiste en ajustar 'un tornillo, un tornillo, un tornillo' (como se muestra en “Tiempos Modernos” de Chaplin), sino la posibilidad de concebir y realizar con las manos propias la forma final de tus productos. Obviamente se trataría de algo más cercano a la manufactura y no a lo industrial, o lo que quieras... Pensar en todas las formas de trabajo, ahí es donde reside la cuestión del homo faber. Y claro, ahí está la actitud de la sospecha generalizada, de no asumir nada como dado... pensar en todas las formas de trabajo, cualificarlas, no desdeñarlas en bloque. Es igual que desarmar, desarmar algo para saber cuál es su magia, pa' que no te hipnotice. Porque si no hacemos eso terminamos en el sonambulismo del consumo y de lo dado, en donde la libertad individual es la libertad de escoger entre Adidas y Calvin Klein: la libertad aparente del mercado. Se estrecha todo a unos niveles tan prosaicos que claro, ya no es libertad, es la cárcel del consumo, la adicción normalizada, ¿no ve?, y cualquier desobediencia tiene su precio. Entonces lo que proponemos es recuperar ese aspecto de la episteme. Porque en el momento de convivir entre las dos... ahí está el chiste! Ahí está el fenómeno emancipador.

Y por el lado de la episteme india, estaría en primer lugar el diálogo con y el reconocimiento de sujetxs no humanos. Eso es absolutamente clave. Cuando voy al mercado y le pido rebaja a la señora que vende papas, no le estoy diciendo 'rebajame', le digo 'ay, esas papitas me están mirando!', le digo, 'me están mirando así' y eso quiere decir que 'a mí nomás vas a tener que vender, casera'. Y entonces, evidentemente, es la papa la que le está diciendo 'pues sí, yo quiero irme en esa bolsa'. Esa relación es muy cotidiana y tiene formas muy fuertes, muy... heavies, digamos, de reconocer la agencia de lo no humano, como cuando un yatiri (un sabio o chamán andino) dialoga con el espíritu de los cerros, ¿ya? Y eso también tiene que ver con que los muertos viven, que es otro elemento clave de la episteme indígena. Los muertos viven, vuelven de su mundo a nuestro mundo, nos protegen o nos castigan, no son seres inertes y olvidables. Por último, está el otro elemento importante que es la comunidad, la comunalidad, la tendencia a crear comunidad, no siempre heredada, no siempre asumida en el marco de lo dado.

FP: Y se trata de pensar en comunidades de afinidad.

Disponible en: https://www.youtube.com/watch?v=IiFDxO7_XIY.

${ }^{9}$ En aymara, es la acción de mantener las hojas de coca en la boca, entre los dientes y las mejillas, para absorber su zumo, ayudándose con la llujt'a, una pasta realizada a base de cenizas vegetales. 
SRC: Esa idea de comunidad de afinidad es para mí fundamental y se relaciona con la raíz anarquista. Pero además se trata de afinidades, digamos... no políticas, propiamente... sino afinidades de gesto. Si hemos dicho que 'descolonizar es hacer', en el Tambo hacemos ese gesto de 'no, no hablaremos de descolonización, más bien haremos, haremos lo posible para acercarnos a esa práctica'. Eso también tiene que ver con una cosa que une ambas dimensiones del trabajo, creo yo, la unión entre el trabajo manual y el trabajo intelectual. Eso es fácil de decir, pero no es tan fácil de hacer. Y te lleva a preguntar y a cuestionar muchas cosas. Por ejemplo, ¿cómo vivirán los que hablan tanto del vivir bien? Ese tipo de preguntas y formaciones culturales, esa brecha entre las palabras y los actos, es para mí un rasgo constitutivo de la sociedad colonial.

Pero ahí viene el tejido ch’ixi entre las dos epistemes: ¿cómo asumir cotidianamente la contradicción entre comunidad y persona individual? El problema no es cómo superar esa contradicción sino cómo convivir con ella, cómo habitar en ella. Para mí esto se hace posible a través de la alegoría: por una poiesis autoconsciente capaz de crear condiciones de pleno respeto a la persona individual sin por ello socavar o mermar la fuerza de lo común. Y es este tipo de comunidad, o de "comunalidad" la que se propone para alimentar nuevas formas de organización, nuevos lenguajes de la politicidad. Una comunidad donde no se trabaje con mayorías y minorías, donde el margen de maniobra sea más grande para el individuo o para la individua en términos de desarrollar lo que cada quien puede como potencia, pero también en términos de elección incluso estética. Eso es una meta descolonizadora bien práctica. El incorporar las voces normalmente disidentes de las mujeres y lxs niñxs, por ejemplo, en esa voz comunitaria. No subalternizarlxs. Y hacer del silencio y del trabajo simplemente callado un modo de comunicación. Incorporar elementos del idioma (aymara), el amuki, por ejemplo, que son los momentos de silencio en el ritual, en el trabajo, en muchos espacios y tiempos. Porque el silencio [Silvia hace unos segundos de silencio, mientras inspira fuerte y me mira] es lo que te permite respirar. Y respirar es parte de este nexo con el chuyma, con el corazón y los pulmones, que es el lugar donde se piensa, donde se piensa de un modo comunitario y memorioso. Entonces, es el papel del pasado en el presente, el hablar un idioma indígena, el hacer comunidad, el diálogo con los muertos, el reconocer sujetos no humanos... podríamos decir que ése es un detalle mínimo de una episteme india. Y yo creo firmemente que estos gestos básicos de sociabilidad son comunes a diversos pueblos indígenas en Abya Yala y quizás en el planeta. Está también el hecho de que hay diversos tipos 
de sujetxs (en el idioma) y a la vez que no hay propiamente sujetos plenamente constituidos: ni seres ni entidades fijas, sino que hay más bien devenires en relación del todo con todo. Bueno, hay una serie de elementos que los estoy desarrollando en mi nuevo libro, pero te advierto que he optado por pensar más a fondo la episteme india por una razón: porque a la otra ya la tenemos incorporada. Aunque como sentido común hay que desmantelarla, hay como una curiosidad mayor por explorar la episteme india para ponerla en la balanza de igual a igual y para que se choquen mejor, pa' que saquen más chispas al chocarse. Y creo que esas chispas: uno, energizan; dos, convierten la frontera de una raya en un tejido. Y la gran metáfora para mí es la wak'a. La wak'a es a la vez el nombre genérico de los lugares sagrados y poderosos del paisaje andino, y el cinturón o faja que usan hombres y mujeres en el trabajo y en los rituales. Es lo que une lo de abajo con lo de arriba y lo que entreteje los opuestos. Es para mí una alegoría que permite convivir entre diferentes manteniendo la radicalidad de la diferencia. Eso sería lo ch 'ixi. O sea, ch'ixi no es la comodidad con la que recibes las aporías, las contradicciones flagrantes que se viven, no es eso. Es más bien la incomodidad y el cuestionamiento que permite sacar todo lo superfluo, la hojarasca que está obstruyendo ese choque y esa energía casi eléctrica, reverberante, que permite convivir y habitar con la contradicción, hacer de ella una especie de visión radiográfica que permita ver las estructuras que subyacen a la superficie. Y eso para mí sería recuperar aquellos otros modos para que convivan sin que el destino sea que uno tenga que ganarle siempre al otro. El ejemplo así más evidente tal vez sea el de repensar el individuo con la comunidad, u otros dualismos: cómo pensar en una espiritualidad materialista o cómo se puede conectar la filosofía con la comida.

FP: Lo que siempre encontré interesante en tus planteos, es que no estás necesariamente tratando de explicar lo ch'ixi. A lo sumo, estás intentando observarlo, explicitarlo. Pero la intención siempre es la de 'conectarse' con eso y hacer de él un método para seguir conociendo, o un método para pelear, para lo que fuera...

SRC: Exacto, se trata digamos de 'extrapolar' las ideas básicas de lo ch 'ixi pero para pensar tu propia realidad, para explorar las contradicciones que observas, las más pertinentes a tu realidad, se trata de extrapolar el 'método'... pero no es un 'citar' un concepto. Ch'ixi es un devenir. Se trata de liberarnos de la esquizofrenia colectiva. Por qué tenemos que siempre estar en las 
disyuntivas de lo uno o de lo otro, somos pura modernidad o pura tradición?. Tal vez somos las dos cosas..., pero las dos cosas no fundidas, porque ese fundido privilegia a un solo lado. Por eso es que lo cholo no termina de ser una identidad ch'ixi, salvo que se asuma como tal, que se asuma como propuesta. Cosa que a pesar de Zavaleta y todos sus exégetas no se ha puesto en evidencia. Para mí la cosa está ahí, hay que profundizarla.

FP: Pensar lo ch'ixi como devenir también habilita nuevas posibilidades para abordar la conexión entre luchas que son, digamos, minoritarias. Pero que justamente por eso se conectan, no? ¿Cuáles son los ejemplos que rescatás para pensar?

SRC: Bueno, las contraferias y las solidaridades que se dan por ejemplo cuando hemos marchado en el TIPNIS todos los grupos anarcos, ch'ixis, o lo que sea... nos unimos en una gran caravana en donde había de todo y de todos lados, de Sucre, de Cochabamba, de La Paz, Teníamos un gran letrero, que me ha encantado, que decía 'somos la etnia Macana' [risas]. Y la macana es el palo, y la macana es la menstruación, y macanear es joder... entonces de algún modo era nuestra gran broma, que nos unía a todos, 'somos los Macana'. No somos Tacanas pero somos Macanas, eso hicieron lxs colectivxs de Cochabamba. Y todos hemos vivido una felicidad total, porque sabíamos que iba a durar poco, pero igual la hemos vivido como una celebración de la fuerza colectiva.

FP: Pero también es necesario, indispensable, reivindicar lo efímero.

SRC: Ay si, absolutamente, absolutamente! Lo transitorio y lo efímero, pero que te deja algo. No habría forma de resistir lo que estamos viviendo si no fuera por la memoria del TIPNIS, la memoria de octubre, la memoria de los esfuerzos que se han hecho en todas las últimas luchas. Esas son semillas, que nos dan energía, que nos han permitido un momento para vislumbrar otra cosa y para vivirla. No se trata de vislumbrarlas para luego intentar vivirlas, sino es el sólo hecho de vivirlas. Eso nos ha dado otra relación con la selva, otra relación con los árboles, otras relaciones con el Brasil. Entonces lo ch'ixi no tiene un nivel de abstracción suficiente como para ser considerado un concepto, porque siempre es como un llamado a superar la primera disyuntiva colonial, que es la de hacer de las palabras un velo. El primer gesto colonial, cuando se encuentra 
el español con el indio, es hacer de la palabra una cosa engañosa. Decir "sí, como oro". Para mí, eso es una gran metáfora. Y el concepto metáfora, la idea de asociar el concepto con la metáfora, es una idea de la Spivak y yo, bueno, como siempre hago con todo, me lo he traído y me lo he extrapolado hacia la metaforización que ya está inscrita en la polisemia del aymara. Y aunque yo no hablo bien el aymara tengo, además de los diccionarios que son una maravilla, la gente aymara hablante con la que interactúo que me da muchas pautas para encontrar hilos. Yo pienso que se puede hacer eso con muchos idiomas; el guaraní es muy hermoso, por ejemplo. Y aunque sea lejano, también es lejano el alemán. Ahora, lxs hijxs de lxs qamiris $^{10}$ aymaras están aprendiendo mandarín para hacer negocios... todo el mundo preparándose para el nuevo round de la globalización. Y no nos ponemos a pensar que lo nuestro está quedando en el desván de trastos viejos, es muy fregado eso. Está muy bien criticar, está muy bien hacer uso de conceptos útiles para poder defenderte, pero es hora de conectar con otras opciones. Que no necesitan ser opciones ni muy espectaculares, ni quiero emblematizar que yo soy muy revolucionaria o alternativa de nada. Si no en cada lugar donde estás tratar de ensanchar el espacio de la autonomía y de la libertad, desde tu lugar, incluso desde la cárcel, nadie te puede gobernar tu pensamiento ni la moral íntima de tus actos.

FP: ¿Es con estos temas que estás trabajando ahora? ¿Vinculados con la sociología de la imagen?

SRC: Es que la sociología de la imagen es un proyecto a medias, es un proyecto en curso. Por más que se ha cristalizado en un libro, es una serie de fragmentos. 'En busca de una episteme propia' es como se llama la segunda parte, que es una búsqueda, que tiene que ver con la memoria, con las sangres que la están habitando, con los entornos y con los paisajes. El otro elemento es traducirnos, es decir, encarnarnos con nuestro entorno de montañas, cerros, lagos, etc. Comprometernos con esos seres que están ahí, cada quien en su entorno. Gozar también de los triunfos que se dan en otros lados. Si le han dado una batalla a Monsanto en Córdoba o si los Sioux le han puesto freno a un oleoducto, todos esos son nuestros triunfos también. Es un nosotros multiplicado, ¿no ve?

Córdoba, Argentina, 16 de septiembre de 2016

\footnotetext{
${ }^{10} \mathrm{El}$ término aymara qamiri puede traducirse como 'rico' o 'próspero' y es utilizado para referir a élites indígenas del altiplano orureño, dedicadas a diferentes ramas del comercio y del intercambio. 


\section{REFERENCIAS}

GARCÍA LINERA, Álvaro. 2014. Identidad Boliviana. Nación, mestizaje y plurinacionalidad. La Paz: Vicepresidencia del Estado plurinacional de Bolivia.

Disponible en: https://www.vicepresidencia.gob.bo/IMG/pdf/identidad_boliviana_.pdf

GUTIÉRREZ FLORES, José Agustín. 2010. "Ese cholo soy yo. Reflexiones sobre la identidad de una mosca". El Colectivo 2, 3: 19-22.

RIVERA CUSICANQUI, Silvia. 2015. Mito y Desarrollo en Bolivia. El giro colonial del gobierno del MAS. La Paz: Plural.

RIVERA CUSICANQUI, Silvia y Virginia AILLÓN SORIA. 2015. "Desde los márgenes. Pensadoras y pensadores bolivianxs de la diáspora”. En Silvia Rivera Cusicanqui y Virginia Aillón Soria (coords). Antología del pensamiento crítico boliviano contemporáneo. Buenos Aires: CLACSO. Pp 13-39.

Disponible en:

https://www.biblioteca.clacso.edu.ar/clacso/se/20151023031022/AntologiaBolivia.pdf.

RIVERA CUSICANQUI, Silvia y El Colectivo (coords). 2010. Principio Potosí Reverso. España: Museo Nacional Centro de Arte Reina Sofía.

RIVERA CUSICANQUI, Silvia y Zulema LEHM ARDAYA [1988] 2014. Lxs artesanxs libertarixs. Buenos Aires: Tinta Limón Ediciones. 